\title{
Newborn Septic Arthritis-A Rare Presentation of Late-Onset Group B Streptococcal Disease: Case Report and Short Review of the Literature
}

\author{
Rahel Schuler, MD ${ }^{1}$ Harald Ehrhardt, MD, $\mathrm{PhD}^{1} \quad$ Klaus-Peter Zimmer, MD, PhD ${ }^{1}$ \\ Daniel Berthold, MD, $\mathrm{PhD}^{2}$ Janina Trauth, $\mathrm{MD}^{3}$ Christian Fölsch, MD, PhD ${ }^{4}$ Markus Waitz, MD ${ }^{1}$
}

${ }^{1}$ Department of General Pediatrics and Neonatology, Justus Liebig University, Giessen, Germany

${ }^{2}$ Department of Diagnostic and Interventional Radiology, University Hospital Giessen, Justus Liebig University, Giessen, Germany

${ }^{3}$ Department of Medicine II, Section of Infectious Diseases, Justus Liebig University, Giessen, Germany

${ }^{4}$ Department of Orthopaedic Surgery, Justus-Liebig-University, Giessen, Germany

AJP Rep 2021;11:e123-e126.

\author{
Address for correspondence Rahel Schuler, MD, Department of \\ General Pediatrics and Neonatology, Justus Liebig University, Giessen \\ 35392, Germany \\ (e-mail: Rahel.schuler@paediat.med.uni-giessen.de).
}

\begin{abstract}
Keywords

- septic arthritis

- newborn

- late-onset sepsis

- group B Streptococcus disease

Group B Streptococcus (GBS) disease is a leading cause of invasive bacterial infections among neonates. We present the case of an 11-day-old neonate with septic arthritis as a rare presentation of late-onset disease (LOD) with a favorable short-term outcome. GBS is a leading cause of neonatal infection. Early-onset disease (EOD) is defined as infection from birth to 6 days of age, while LOD occurs from 7 days to approximately 3 months of age. EOD is acquired through vertical transmission and can be reduced through application of intrapartum antibiotic prophylaxis (IAP). LOD can be acquired from the mother or from environmental sources, unlikely to be prevented by IAP. The most common presentation of EOD is bacteremia (83\%), pneumonia (9\%), and meningitis (7\%). While the clinical picture in both EOD and LOD frequently resembles in LOD hamatogenous spreading may predispose neonates to present with uncommon organ manifestation other than the classic systemic signs of sepsis, for example, septic arthritis. Herein, we report on the management and outcome of a term neonate with late onset GqBS bacteremia and subtle clinical symptoms of septic monoarthritis.
\end{abstract}

\section{Case Presentation}

We report of a male neonate, born to a 29-year-old primigravida at $40^{2 / 7}$ weeks of gestation, after an uneventful pregnancy and normal prenatal diagnostic screening. The uncomplicated spontaneous vaginal delivery took place in a regional hospital. Group B Streptococcus (GBS) screening of the mother was negative. The birth weight was 4,574 g; Apgar's values were 10 and 10 at 5 and 10 minutes, respectively. The healthy infant was discharged 3 days after birth.

On day of life 11, the mother presented the infant to our outpatient department (OPD). Since the evening before the infant did not extend the left leg, with the affected limb kept in a continuously flexed position. Significant pain perceptions received

April 13, 2021

accepted after revision

May 10, 2021
DOI https://doi.org/

10.1055/s-0041-1735633.

ISSN 2157-6998.

\footnotetext{
(C) 2021. The Author(s).

This is an open access article published by Thieme under the terms of the Creative Commons Attribution-NonDerivative-NonCommercial-License, permitting copying and reproduction so long as the original work is given appropriate credit. Contents may not be used for commercial purposes, or adapted, remixed, transformed or built upon. (https://creativecommons.org/ licenses/by-nc-nd/4.0/) Thieme Medical Publishers, Inc., 333 Seventh Avenue, 18th Floor, New York, NY 10001, USA
} 
were reported whenever the leg was mobilized. The infant was otherwise healthy, feeding well; there was no fever and no known trauma.

Upon examination, the infant was alert, the body temperature was $37.8^{\circ} \mathrm{C}$, the infant held the left leg in a flexed protective posture and resisted active extension of the knee joint. There was tenderness on palpation within the knee joint region without any redness or swelling. Clinically, no presence of fluid in the knee joint could be elicited. The physical examination was otherwise normal.

Laboratory results showed a slightly elevated C-reactive protein (CRP) of $21 \mathrm{mg} / \mathrm{L}$ and a normal white blood cell count without a left shift in the white blood cell differentiation. A blood culture was obtained. Sonography of the hip and knee was normal with no detectable joint effusions. After admission, antibiotic treatment with ampicillin $(150 \mathrm{mg} / \mathrm{kg} /$ day $)$ and gentamicin $(4 \mathrm{mg} / \mathrm{kg} /$ day) was initiated due to the slightly elevated CRP and suspected osteomyelitis or arthritis. The magnetic resonance imaging (MRI) on the next day showed a small effusion within the left knee joint, suggestive of a local inflammatory process not affecting the extraarticular bone tissue and most likely considered as septic arthritis (-Fig. 1). The ultrasound scan one day later eventually confirmed a small effusion. After GBS isolation in the blood culture (positive after 6.2 hours) antibiotic treatment was switched to intravenous penicillin $\mathrm{G}$ (300,000 units $/ \mathrm{kg} /$ day divided in doses every 8 hours) and continued for

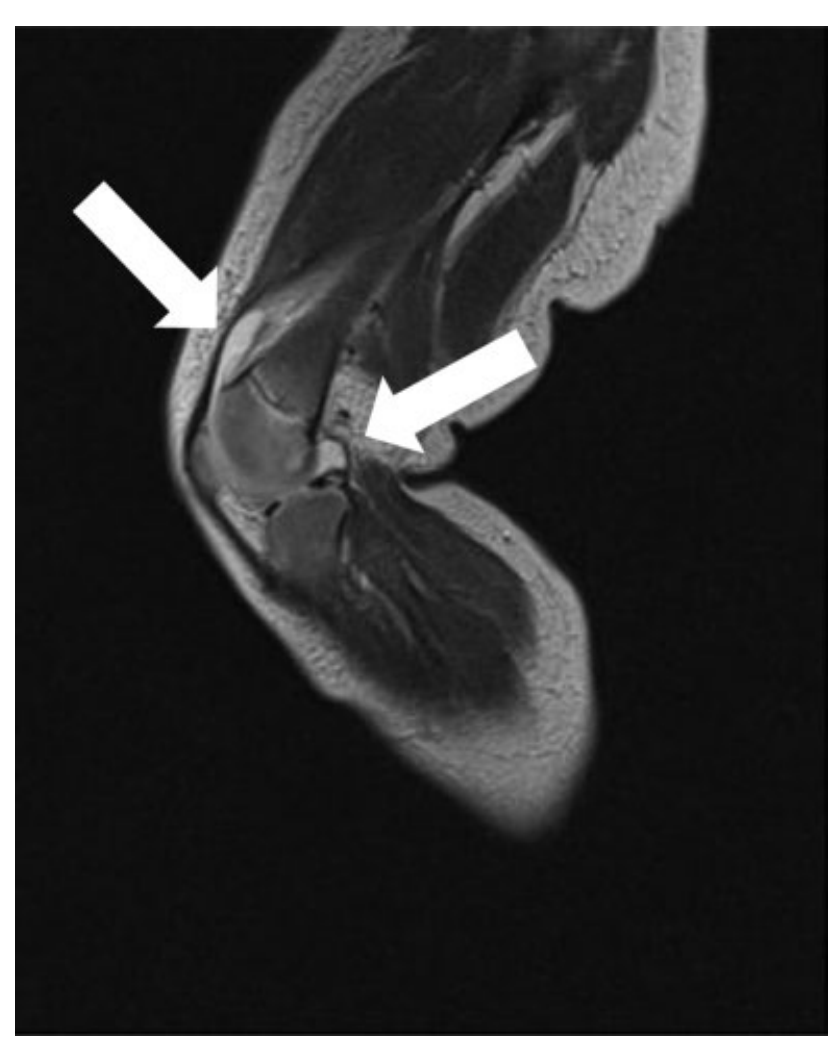

Fig. 1 MRI (1.5 T), surface coil. T2 Turbo Spin Echo coronal $3 \mathrm{~mm}$. Left knee: small amount of Joint effusion in the suprapatellar and posterior recess (arrows). No bone affection was noted. MRI, magnetic resonance imaging.

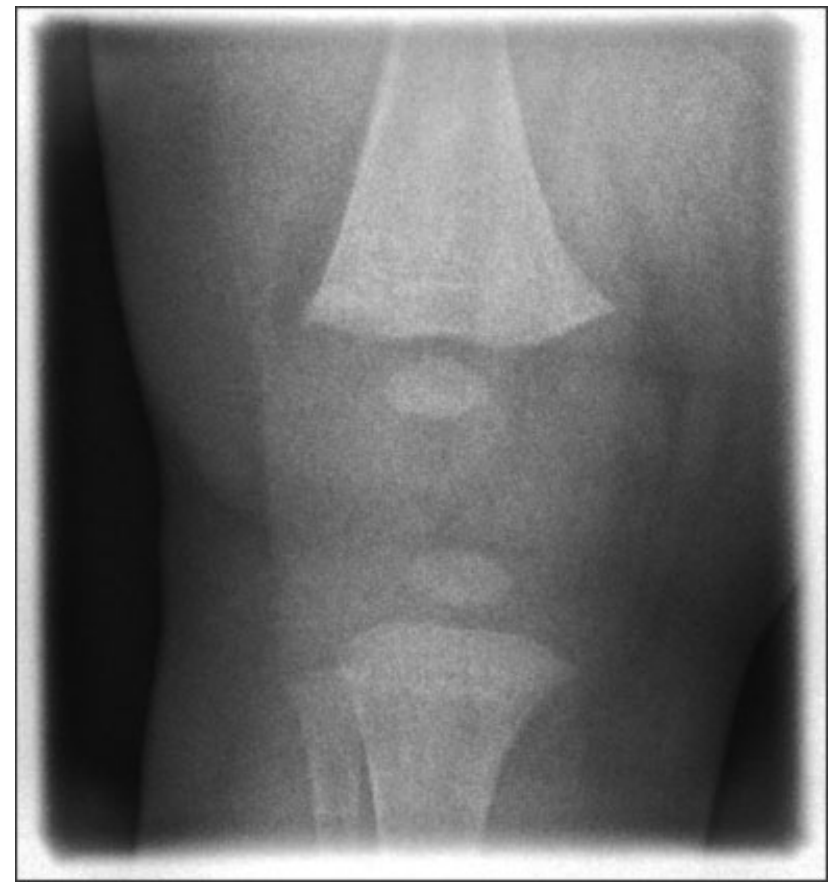

Fig. 2 Anterior-posterior X-ray of the left knee 33 days after the MRI examination. MRI, magnetic resonance imaging.

21 days. Despite the positive blood culture, lumbar puncture was omitted due to lacking clinical signs of meningitis.

Forty-eight hours after the initiation of antibiotic treatment, the CRP level decreased to $11 \mathrm{mg} / \mathrm{L}$ and the infant started to move the affected leg actively. After 7 days, there was no more pain during active or passive movement of the leg.

Repeated GBS swabs of the mother and expressed breast milk yielded a negative result.

Before discontinuation of the antibiotic therapy, ultrasound of the knee was repeated and an X-ray was performed, both inconspicuous. Results of the clinical follow-up at the OPD 14 days after discharge indicated fully functional recovery of the left knee joint and ultrasound and X-ray imaging of the affected left knee ( - Fig. 2 ) remained unremarkable.

\section{Discussion}

Septic arthritis is a rare presentation of group B streptococcal late-onset disease (LOD) and contributes to only 4\% (4/100) in an Italian cohort $(2003-2010)^{1} ; 0.73 \%(2 / 274)$ in a Japanese cohort (2011-2015), ${ }^{2}$ and no reported case (0/1036) in a cohort in the United States (1995-2005) ${ }^{3}$ of all cases of LOD.

Clinical signs of septic arthritis include pseudoparesis, local swelling, redness, and fever. ${ }^{4,5}$ Our patient exclusively showed signs of pseudoparesis and local tenderness on palpation. The mildly elevated CRP pointed toward an inflammatory process, while the white blood cell count was normal. This, however, is in contrast to a case series from India, where infants diagnosed with septic arthritis universally had leucocytosis. ${ }^{6}$

Due to the described subtle clinical signs along with the results of the MRI and the positive blood culture, we suspected septic arthritis in our case. As the onset of symptoms 
in our patient just occurred the evening before the presentation in the OPD and the clinical evaluation and laboratory results were unspecific, the early diagnosis of septic arthritis might have been missed without additional imaging investigations, that is, MRI. Particularly, the use of the ultrasound was unreliable in the early course of the disease in our case and might have led to a missed diagnosis of septic arthritis if considered as a sole imaging investigation. Umadevi and colleagues reported on a similar case of a term neonate with septic arthritis of the elbow which was initially diagnosed as joint dislocation. The ultrasound in this case, in contrast to our patient, showed significant effusions indicating a more progressive state of the disease as compared with our case, and the culture of the effusion aspirate finally lead to the diagnosis of GBS-induced septic arthritis in their case. ${ }^{7}$ As GBS was cultured in the blood sample, we speculate that this is the most likely pathogen and cause for the arthritis in our patient, although we did not confirm this through a culture of the effusion aspirate. The decision to omit needle aspiration was mainly based on the insignificant small effusion and in consent with the pediatric orthopaedic consultant. It should be mentioned that septic arthritis and osteomyelitis often occur concomitantly in the newborn infant due to the unique vascular anatomy of the neonate with transphyseal vessels freely communicating between epiphysis and metaphysis. ${ }^{8,9}$ Although the diagnosis of osteomyelitis was unlikely based on the results of the initial MRI, we cannot definitely exclude minor osteomyelitis.

Early diagnosis and immediate proper treatment are important to avoid long-term impairment including joint destruction, deformity of limbs, and growth failure. ${ }^{10}$

Surgical drainage of the affected joint is commonly recommended to obtain biological samples and decrease intraarticular pressure. In our patient, the effusion was small and there were no signs of increased intra-articular pressure. Although surgical drainage could have provided a definitive diagnosis that we decided against it, we did not expect drainage of the small effusion to improve the clinical outcome. In a retrospective analysis of 52 cases of neonatal septic arthritis, surgical intervention did not improve the outcome ${ }^{4}$ but randomized trials are lacking.

Regardless of any surgical interventions, intravenous antibiotic treatment for neonatal septic arthritis is additionally required and the recommended duration of treatment is currently 14 to 21 days. ${ }^{11}$ This is mainly based on expert opinion or local guidelines. However, a recent retrospective analysis reported on the safe use of shorter antibiotic courses for uncomplicated GBS bacteraemia. ${ }^{12}$ Intravenous treatment of septic arthritis or acute osteomyelitis in children older than 3 months of age for only 2 to 4 days, followed by a course of oral antibiotics for 7 to 10 days, was shown to be as effective as a course of 30 days of treatment ( 3 days intravenous and 27 days oral) in two randomized trials from Finland. ${ }^{13,14}$ Although there is a trend toward shorter courses of antibiotic treatment in older patients and uncomplicated LOD, randomized data on such an approach for neonates with complicated LOD are lacking. In a systematic review regarding oral antibiotics for neonatal infections, the authors conclude that although promising results are available, well-designed studies in high-income countries are lacking and required before this approach can definitely be recommended for neonates. ${ }^{15}$ In our case, a 21-day course of intravenous antibiotics resulted in a favorable short-term clinical outcome and the results of the follow-up 35 days after the diagnosis confirmed no signs of local or systemic inflammatory relapse.

\section{Conclusion}

In neonates with proven late-onset GBS bacteremia, clinical awareness including a thorough physical examination of the infant and MRI scan in uncertain cases is recommended to exclude or confirm local bone manifestations if suspected. As a consequence of the diagnosis of septic arthritis associated with GBS LOD, prolonged antibiotic treatment is warranted in addition to surgical intervention in selected cases to avoid long-term functional sequelae. The early diagnosis of septic arthritis and timely initiation of antibiotic treatment in our case may have contributed to the favorable short-term outcome.

\section{Conflict of Interest \\ None declared.}

\section{References}

1 Berardi A, Rossi C, Lugli L, et al; GBS Prevention Working Group, Emilia-Romagna. Group B streptococcus late-onset disease: 2003-2010. Pediatrics 2013;131(02):e361-e368

2 Matsubara K, Hoshina K, Kondo M, et al. Group B streptococcal disease in infants in the first year of life: a nationwide surveillance study in Japan, 2011-2015. Infection 2017;45(04):449-458

3 Phares CR, Lynfield R, Farley MM, et al; Active Bacterial Core surveillance/Emerging Infections Program Network. Epidemiology of invasive group B streptococcal disease in the United States, 1999-2005. JAMA 2008;299(17):2056-2065

4 Li Y, Zhou Q, Liu Y, et al. Delayed treatment of septic arthritis in the neonate: a review of 52 cases. Medicine (Baltimore) 2016;95 (51):e5682

5 Pittard WB III, Thullen JD, Fanaroff AA. Neonatal septic arthritis. J Pediatr 1976;88(4 pt 1):621-624

6 Sreenivas T, Nataraj AR, Kumar A, Menon J. Neonatal septic arthritis in a tertiary care hospital: a descriptive study. Eur J Orthop Surg Traumatol 2016;26(05):477-481

7 Umadevi S, Kali A, Sreenivasan S, Pramodhini S, Charles MV. Septic arthritis caused by group A Streptococcus in newborn: an unusual presentation. J Clin Diagn Res 2013;7(06):1143-1144

8 Offiah AC. Acute osteomyelitis, septic arthritis and discitis: differences between neonates and older children. Eur J Radiol 2006;60 (02):221-232

9 McPherson DM. Osteomyelitis in the neonate. Neonatal Netw 2002;21(01):9-22

10 Castellazzi L, Mantero M, Esposito S. Update on the management of pediatric acute osteomyelitis and septic arthritis. Int J Mol Sci 2016;17(06):E855

11 Kimberlin DW, Brady MT, Jackson MA, eds. Red Book: 2018-2021 Report of the Committee on Infectious Diseases. 31st ed. Itasca, IL: American Academy of Pediatrics; 2018:762

12 Coon ER, Srivastava R, Stoddard G, Wilkes J, Pavia AT, Shah SS. Shortened IV antibiotic course for uncomplicated, late-onset group B streptococcal bacteremia. Pediatrics 2018;142(05): e20180345 
e126 Late-Onset Group B Streptococcal Disease in a Neonate Schuler et al.

13 Peltola H, Pääkkönen M, Kallio P, Kallio MJOsteomyelitis-Septic Arthritis (OM-SA) Study Group. Prospective, randomized trial of 10 days versus 30 days of antimicrobial treatment, including a short-term course of parenteral therapy, for childhood septic arthritis. Clin Infect Dis 2009;48(09):1201-1210

14 Peltola H, Pääkkönen M, Kallio P, Kallio MJOsteomyelitis-Septic Arthritis Study Group. Short- versus long-term antimicrobial treatment for acute hematogenous osteomyelitis of childhood: prospective, randomized trial on 131 culture-positive cases. Pediatr Infect Dis J 2010;29(12):1123-1128

15 Keij FM, Kornelisse RF, Hartwig NG, Reiss IKM, Allegaert K, Tramper-Stranders GA. Oral antibiotics for neonatal infections: a systematic review and meta-analysis. J Antimicrob Chemother 2019;74(11):3150-3161 